\title{
Pulsed fluoroscopy in retrograde urethrograms
}

\author{
Hazem Elmansy ${ }^{1}$, Waleed Shabana ${ }^{1}$, Radu Rozenberg ${ }^{2}$, Abdulrahman Ahmad ${ }^{1}$, Ahmed Kotb ${ }^{1}$, \\ Amer Al Aref ${ }^{2}$, Walid Shahrour ${ }^{1}$ \\ ${ }^{1}$ Department of Urology and ${ }^{2}$ Radiology, Northern Ontario School of Medicine, Thunder Bay, ON, Canada.
}

\begin{abstract}
Summary Objectives: Retrograde urethrogram (RUG) is one of the corner stones for the reconstructive urologist. With hundreds of RUGs being performed yearly in busy reconstructive center, the concern for radiation exposure to the patient and the medical personnel becomes important. We propose the use of pulsed fluoroscopy to decrease the radiation exposure for patient and medical personnel.

Methods: Patients presenting to our center with urethral strictures between March 2016 and March 2019 were included in our study. The fluoroscopy machine was set for pulsed fluoroscopy at a setting of 4 pulses per second. Patient information including demographics, pre-operative diagnosis, Intra-op findings, and fluoroscopy time were recorded. RUG was performed to localize the stricture pre-operatively and post-operatively. Results: A total of 185 RUG were performed between March 2016 and March 2019. The median age was 63 (14-81).

The remaining 154 RUG had 77 performed pre-operatively and 77 performed post-operatively. Pathology was identified in 77 patients. Intra-operative confirmation of pre-operative finding was found in 76 patients (98.7\%). Median fluoroscopy time was found to be 2.43 seconds $(0.5 \mathrm{sec}-6.5 \mathrm{sec})$.

Conclusions: Pulsed fluoroscopy reduces the radiation exposure in RUG without a reduction in the diagnostic capacity of the test. Reduction of fluoroscopy can have beneficial cumulative effect as per the ALARA principle for patients and medical personnel. Further studies with randomized control trials could be of great benefit.
\end{abstract}

KEY WORDS: Urethrogram; Pulsed fluoroscopy.

Submitted 19 March 2021; Accepted 21 April 2021

\section{INTRODUCTION}

Retrograde urethrography (RUG) and voiding cystourethrography (VCUG) are the modalities of choice for imaging the male urethra. First, RUG is performed to visualize the adequately distended anterior urethra, and VCUG is then performed to properly evaluate the posterior urethra. Urethrography is a dynamic imaging modality that should be done by an expert urologist to assure the accuracy of the technique and the correct interpretation thereafter $(1,2)$. Reducing the fluoroscopic exposure without compromising the image quality was always the first priority for manufacturers as well as the surgeons, hence the invention and implementation of the ALARA principle "As Low As Reasonably Achievable" in 1990 (3). The ALARA principle has 3 factors, time, distance, and shielding. There are a variety of ways to reduce fluoroscopy time during interventional procedures; some are methodological and others involve taking advantages of technical features present in modern equipment such as intermittent fluoroscopy, removal of grid, last image hold, electric collimation, dose spreading, adjustment of beam quality, image magnification, dose level settings, and pulsed fluoroscopy (4).

The idea behind pulsed fluoroscopy is that modern gridcontrolled x-ray tubes have a grid placed between the cathode and the anode which allows pulses of fluoroscopy to leave the tube at a rate between 1 and 30 frames per second (FPS).

Thus, radiation no longer enters a patient continuously, but rather in a series of short x-rays flashes. When we use fluoroscopy at 30 FPS it is called continuous fluoroscopy; on the other hand, Aufrichtig et al. (5) defined pulsed fluoroscopy as 15 FPS or less. Each fluoroscopy unit could be manually set as pulsed fluoroscopy (PF) with refresh rates of 15,8 , or even 4 FPS. Using phantom models, PF at rates of $15,10,7.5$, and 3.75 FPS were associated with radiation reduction by $22 \%, 38 \%, 49 \%$, and $87 \%$, respectively $(6,7)$. Although using pulsed fluoroscopy appears promising in reducing radiation, it always has a potential penalty of a decrease in image quality. On a real-time fluoroscopic image, low pulse rate makes image becomes more noisy or grainy. Moreover, with very slow pulse rates, motion such as swallowing, peristalsis and heart beating becomes jerky (choppy). To overcome this obstacle, manufacturers increase the milliamperage settings to achieve a similar visual appearance $(8,9)$. During retrograde urethrography, using pulsed fluoroscopy is ideal because there is minimal patient movement and we use the real time imaging to delineate the urethra with better identification of the urethral pathology (10).

The primary objective is to identify if the use pulsed fluoroscopy mode during retrograde urethrogram to minimize radiation exposure for both the operator and the patient.

The secondary objective is to assess whether using 4 frames per seconds (FPS) in pulsed mode is sufficient without the need to increase the number of FPS. We will also assess the image quality of the study and the concordance between the image and the intraoperative finding during diagnostic cysto-urethroscopy.

No conflict of interest declared. 


\section{Methods}

Patients presenting to our center with urethral strictures between March 2016 and March 2019 were included in our study. RUGs were performed by a single urologist. The fluoroscopy machine was set for pulsed fluoroscopy at a setting of 4 pulses per second. The same technique of RUG was used in every test. The urologist controlled the pedal for fluoroscopy. Patient information including demographics, pre-operative diagnosis, fluoroscopy time, stricture location and length, intra-op findings, and intraop cystoscopy were recorded. RUG was performed to localize the stricture pre-operatively. If there is no identification of a stricture, cystoscopy is performed to confirm the negative findings. It was also performed in select patients post-operatively with cystoscopy to confirm the findings. The data was collected retrospectively after ethics approval. Patients that had complex stricture disease requiring fluoroscopic manipulation were excluded.

\section{Results}

A total of 185 RUG were performed between March 2016 and March 2019. The median age was 63 (14-81). There were 20 RUG that did not show a stricture, and this was confirmed by cystoscopy in the same setting. There were 11 patients that were excluded as they had complex stricture disease requiring fluoroscopic manipulation. The remaining 154 RUG had 77 performed preoperatively and 77 performed post-operatively.

Pathology was identified in 77 patients. Intra-operative confirmation of pre-operative finding was found in 76 patients $(98.7 \%)$. There were no recorded complications from the RUG. Strictures locations and demographics were recorded in Table 1. Median fluoroscopy time was found to be 2.43 seconds $(0.5 \mathrm{sec}-6.5 \mathrm{sec})$.

\section{Discussion}

RUG is a fundamental test for reconstructive urethral surgery. In the high-volume centers, multiple RUGs are being performed on a daily basis. As per the ALARA principle, we would always strive to decrease the amount of radiation used. This can help the medical personnel that are exposed on daily basis to radiation. In addition,

\section{Table 1.}

Study population and pulsed urethrogram data.

\begin{tabular}{|l|c|}
\hline Number of patients & 77 \\
\hline Total number of RGU & 185 \\
\hline Negative RUG (excluded) & 20 \\
\hline Pre and post-operative RUG & 154 \\
\hline Complex RUG (excluded) & 11 \\
\hline Median age (IQR) years & $63(58-71)$ \\
\hline Median fluoroscopy time seconds & $2.43(0.5-6.5)$ \\
\hline Intra-operative confirmation of structure & $76(98.7 \%)$ \\
\hline Stricture location: & $21(27.2 \%)$ \\
\hline Penile n (\%) & $42(54.5 \%)$ \\
\hline Bulbar n (\%) & $14(18.1 \%)$ \\
\hline Pan-urethral $n(\%)$ & \\
\hline
\end{tabular}

with increased dependence on radiological assessments, we would aim at decreasing the accumulated radiation for patients over the years. In our current study, we noted that the fluoroscopy time has a median of 2.43 seconds. According to the studies on phantom models, this might mean an $87 \%$ reduction in the amount of radiation $(6,7)$. We do not have previous results with continuous fluoroscopy or higher pulse rate as this was adopted from the beginning. The usual extrapolation of the data would be that the amount of fluoroscopy would be more than doubled with the higher pulse rate. The use of the fluoroscopy time is not always the best indicator for the cumulative dose compared to the use of the air kerma area product or dose area product (DAP). In our study we used the fluoroscopy time as it is a simple way to convey the result and it is one of the indicators for the reduction in the dose.

The exclusion of the complicated cases that required fluoroscopic manipulation was due to the fact that they were not representative of the usual fluoroscopy time used in regular diagnostic procedure. This was not due to the image quality being poorer rather than it would bring the average fluoroscopy time to be higher than usual expected. This should not exclude the use of low pulse fluoroscopy in those cases as it would mean lower radiation dose for patients and personnel specially when maneuvers are needed and longer fluoroscopy would be used. The only case where the RUG was not diagnostic entirely because of improper opacification of the distal urethra during the RUG and not because of reduction of the image quality. This was identified during surgery where the intra-operative finding showed the stricture to be extending all the way to the distal urethra. The comparison between the intra-operative findings and the RUG findings did not show reduction in the diagnostic capacity or the capability of pre-operative planning for the surgery.

One of the downsides of the lower pulse rate is the lower quality of the image. The image becomes grainy in appearance and it takes some time to get used to that. This grainy appearance does not affect the image contrast or the ability to diagnose stricture as shown in our study. Another downside is the noticeable delay between pressing the pedal and the appearance of the image on the screen. This delay can be a matter of seconds or milli seconds and it does not usually affect the outcomes.

This would be apparent when the urologist is performing manipulation under fluoroscopy. Since the RUG does not require manipulation, this delay is acceptable for the reduction of the radiation exposure.

Another method to decrease radiation exposure is by having the doctor, residents and fellows taking radiation-safety programs. Gendelberg et al. (11) has shown that radiation-safety programs decrease the radiation emission and usage after the program was taken by the residents.

While some might argue that they use just one image, it is still using the usual frames of the machine. Many machines are set on rates of 15 FPS while newer machines are set at 8 FPS which would be a lower FPS used. The regular continuous fluoroscopy is 30 FPS which would mean more than 7 times the number of frames needed for one image. 
There are methods to decrease the fluoroscopy time and radiation exposure in general that can be used. One can bring the part to be examined (in our case, the urethra) as close as possible to the receiving end of the C-arm or the image intensifier. Using the last image option if we require more than one image can also reduce the radiation exposure.

Our study is a retrospective one with the known limitations of retrospective studies. Randomized multi-institutional trial can prove beneficial in such situation.

\section{Conclusions}

Pulsed fluoroscopy reduces the radiation exposure in RUG without a reduction in the diagnostic capacity of the test. Reduction of fluoroscopy can have beneficial cumulative effect as per the ALARA principle for patients and medical personnel. Further studies with randomized control trials could be of great benefit.

\section{REFERENCES}

1. Pavlica P, Barozzi L, Menchi I. Imaging of male urethra. Eur Radiol 2003; 13:1583-1596.

2. Kawashima A, Sandler CM, Wasserman NF, et. al. Imaging of urethral disease: a pictorial review. RadioGraphics 2004; 24(suppl 1): S195-S216.
3. European ALARA Network Workshop, "Experience and new Developments in implementing ALARA in Occupational, Patient and Public Exposures", Prague, Czech Republic, 12-15 September 2006 , proceedings available on www.eu-alara.net.

4. Vehmas $T$, et al. Hawthorne effect. Shortening of fluoroscopy times during radiation measurement studies. Br J Radiol. 1997; 70:10531055 .

5. Aufrichtig R, Xue P, Thomas CW, et al. Perceptual comparison of pulsed and continuous fluoroscopy. Med Phys. 1994; 21:245-256.

6. Cohen M. Optimizing the use of pulsed fluoroscopy to reduce radiation exposure to children. Am Coll Radiol. 2008; 5:205-209.

7. Lederman HM, Khademian ZP, Felice M, et al. Dose reduction fluoroscopy in pediatrics. Pediatr Radiol. 2002; 32:844-848.

8. Brown PH, Thomas RD, Silberberg PJ, et al. Optimization of a fluoroscopeto reduce radiation exposure in pediatric imaging. Pediatr Radiol. 2000; 30:229-235.

9. Schueler BA, Julsrud PR, Gray JE, et al. Radiation exposure and efficacy of exposure-reduction techniques during cardiac catheterization in children. AJR Am J Roentgenol. 1994; 162:173-7.

10. Ward VL, Strauss KJ, Barnewolt CE, et al. Pediatric radiation exposure and effective dose reduction during voiding cystourethrography. Radiology, 2008; 249:1002-1009.

11. Gendelberg D, Hennrikus W, Slough J et al. A radiation safety training program results in reduced radiation exposure for orthopaedic residents using the mini C-arm. Clin Orthop Relat Res. 2016; 474:580-4.

\author{
Correspondence \\ Hazem Elmansy, MD \\ hazem.mansy@rocketmail.com \\ Waleed Shabana, MD \\ waleed.shabana@gmail.com \\ Abdulrahman Ahmad, MD \\ dr.aaa.186@gmail.com \\ Ahmed Kotb, MD \\ kotba@tbh.net \\ Walid Shahrour, MD \\ walid.shahrour@gmail.com \\ Department of Urology, Northern Ontario School of Medicine, \\ Thunder Bay, ON (Canada) \\ Radu Rozenberg, MD \\ rozenber@tbh.net \\ Amer Al Aref, MD \\ alarefa@tbh.net \\ Department of Radiology, Northern Ontario School of Medicine, \\ 980 Oliver Road, Thunder Bay, ON (Canada)
}

